\title{
Cytogenetic Study of Spontaneous Abortions in the Western part of Romania
}

\author{
SIMONA FARCAS ${ }^{1 \#, ~ N I C O L E T A ~ A N D R E E S C U 1 \# *, ~ D A N I E L A ~ A M Z A R ~}{ }^{2 \#, ~ L A V I N I A ~ S T E L E A ~}{ }^{3}$, ALEXANDRA MIHAILESCU1, \\ ANDREEA DOBRESCU ${ }^{1}$, NOEMI MESZAROS ${ }^{4}$ \\ ${ }^{1}$ Victor Babes University of Medicine and Pharmacy, Discipline of Genetics, 2 Eftimie Murgu Sq., 300041, Timisoara, Romania \\ 2 Victor Babes University of Medicine and Pharmacy, Department of Endocrinology, 2 Eftimie Murgu Sq., 300041, Timisoara, \\ Romania \\ ${ }^{3}$ Victor Babes University of Medicine and Pharmacy, Department of Obstetrics-Gynecology, 2 Eftimie Murgu Sq., 300041, Timisoara, \\ Romania \\ ${ }^{4}$ Vasile Goldis University, Faculty of Medicine, Life Science Department, 94 Revolutiei Blvd., 310025, Arad, Romania
}

\begin{abstract}
Regarding the rate of chromosomal abnormalities in spontaneous abortions, the data varies between 50 and $80 \%$ for first trimester miscarriages but there are several factors that may influence the rate of chromosomal aberrations as the selection bias of the cases for which cytogenetic analysis is done, the gestational age distribution, the maternal age distribution, the tissue samples analyzed, the expertise in cytogenetic methods. The aim of the research is to establish the incidence of chromosomal aberration in our study lot for the patients from the Western part of Romania, as there are no studies available for this specific population. In the present study, patients were included with pregnancy loss between 4 to 14 weeks of gestation for whom cytogenetic evaluation of the fetus was done in the University of Medicine and Pharmacy Timisoara. Of all results, $48.57 \%$ (34 out of 70 ) aborted fetuses had an abnormal karyotype. The gestational age of the aborted fetuses varied between 5 and 14 weeks of gestation, with a mean of 8.95 weeks of gestation. A higher incidence of chromosomal anomalies was observed in the group including patients $\geq 35$ years as compared with the group patients aged under 35 years old (55.5\% vs $45.5 \%)$. The results of cytogenetic analysis of first trimester miscarriage is an important tool that can enhance diagnostic evaluation, and represent a critical information for counselling infertile couples. Post-miscarriage genetic and psychological counselling and appropriate treatment are crucial for the prevention of high psychological distress, psychiatric symptoms and psychopathology in these patients.
\end{abstract}

Keywords: cytogenetic analysis, spontaneous abortions, infertility

It is documented that about 15 to 25 percent of the pregnancies ended up in a spontaneous abortion, while two consecutive pregnancies loss occurs in less than $5 \%$ of the cases and $1 \%$ of the women will have more than 2 recurrent abortions [ $[1,2]$. The majority of the miscarriages are produced between eight and 12 weeks of gestation [3]. In 50 to $70 \%$ of miscarriages a chromosome aberration will be found [4-6]. In about $99 \%$ of pregnancies with chromosome anomalies a miscarriage will be registered [7]. Currently, it is recommended that the evaluation of the patient should begin after two pregnancy losses, and not waiting until the third miscarriage [8-11]. The cytogenetic analysis of the aborted fetus is necessary in order to establish the cause of pregnancy termination and for an adequate genetic counseling of the couple [12-14]. For the cytogenetic evaluation, direct or semi-direct analysis of chorionic villi is routinely used $[5,10,11]$.

It has been further stated that miscarriages, whether sporadic or recurrent, often result in high levels of emotional distress. Miscarriages are a traumatic experience with some degree of psychological implication. Nearly $40 \%$ of women who experience a miscarriage become symptomatic for depression and/or anxiety [15-17]. In a majority of the patients, symptoms persist for 1 to 3 years, quality of life and further pregnancies being affected. Women mostly affected would be those with poor coping and resilience skills [17].

The patients and their partner usually need psychological counselling and psychotherapy in order to reduce the level of emotional distress and to prevent the further psychopathologic impact and development [15, 16]. The approach must be personalized and the whole intervention should be in an ethical, non-stigmatizing frame. The patients can benefit from psychological counselling, cognitive behavioral or systemic psychotherapy, also cognitive restructuring techniques, breathing and relaxation control techniques.

If the patients need medication as well, in order to reduce the distress and anxiety or an eventual posttraumatic stress disorder, the approach should be personalized. If needed, when prescribing medication, the pharmacological intervention should be carefully chosen in function of the psychological profile of the patient, in order to prevent potential adverse events and for improved quality of life [15].

We present here the cytogenetic findings in a series of 70 first trimester pregnancy losses. The aim of the research is to establish the incidence of chromosomal aberration in our study lot for the patients from the Western part of Romania, as there are no studies available for this specific population.

\section{Experimental part}

Patients, materials and methods

In the present study, patients with pregnancy loss between 4 to 14 weeks of gestation for whom cytogenetic evaluation of the fetus was done in the Cytogenetic Laboratory of the University of Medicine and Pharmacy Timisoara, were included. In the present research, 70 patients with miscarriages, aged between 19 and 41 years 
old, were included. Overall mean maternal age was 30.31 years.

The inclusion criteria in the study group were: cases of fetal deaths and abortions, recurrent abortions (more than 2), abortion in couple with known chromosomal aberration. Exclusion criteria were: interruptions of the pregnancy on patient request and patients refusal of cytogenetic evaluation of the fetus. The diagnosis of miscarriage was established on standard ultrasound screening. Patients diagnosed with pregnancy termination were informed about the possibility of having a cytogenetic evaluation of the chorionic villi. For evaluation of the gestational age, is was used the date of the last menstrual period and ultrasound measurements.

\section{Specimen preparation and karyotyping}

The chorionic villi were obtained from the products of conception after the curettage of uterine cavity and placed transport medium. The samples were sentfor karyotyping at the Cytogenetic Lab, where by using the stereomicroscope the chorionic villi were separated from the maternal decidua and blood clots. Metaphase spreads were obtained using the laboratory protocol for short-term culture of chorionic villi (CV) [10]. Chorionic villi were cleaned in Dulbecco's Modified Eagle Medium (Life Technologies). Cells were cultured in three flasks in Chang Medium D (Irvine Scientific). After 5-6 days, cells were harvest according the standard protocol for G-band chromosome analysis.

For each case at least 10 metaphases were analyzed at a 400-band level, when the karyotype was abnormal or normal male. In case of a normal female Karyotype at 20 cells were evaluated to search for a second cell line.

\section{Statistical analysis}

Descriptive statistics are outlined for all variables assessed as mean and standard deviation (SD) for continuous variables and as percentages for categorical variables. Statistical analysis was performed using MedCalc v 18 (MedCalc Software, Ostend, Belgium. The Student's t-test in conjunction with the chi-square test were used to verify the significance between variables. The values were considered significant if $p<0.05$.

\section{Results and discussions}

The available data included the cytogenetic analysis of 70 miscarriages among patients aged between 19 and 41 years old. Overall mean maternal age was 30.31 years (mean deviation Standard 5.67; Confidence Interval (Cl) 99\%: [28.9609-31.6677]), With 52 (74.28\%) women under the age of 35 and $18(25.72 \%)$ between 35 and 41 years' old (fig. 1).

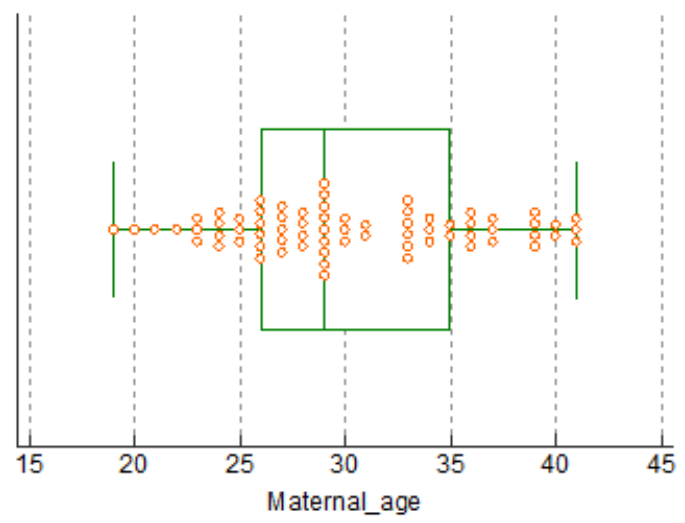

Fig. 1. Maternal age distribution in the study lot
The gestational age of the aborted fetuses varied between 5 and 14 weeks of gestation, with a mean of 8.95 weeks of gestation (mean standard deviation 2.25 Confidence Interval (Cl) 99\%: [8.4195-9.4948]) (fig. 2).

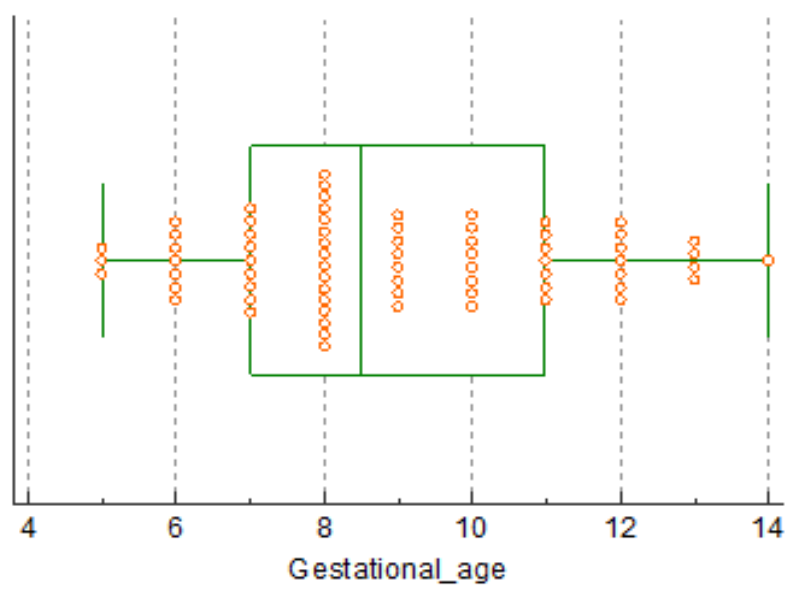

Fig. 2. Gestational age distribution in the study lot

Of all results, $48.57 \%$ ( 34 out of 70 ) aborted fetuses had an abnormal karyotype (fig. 3).

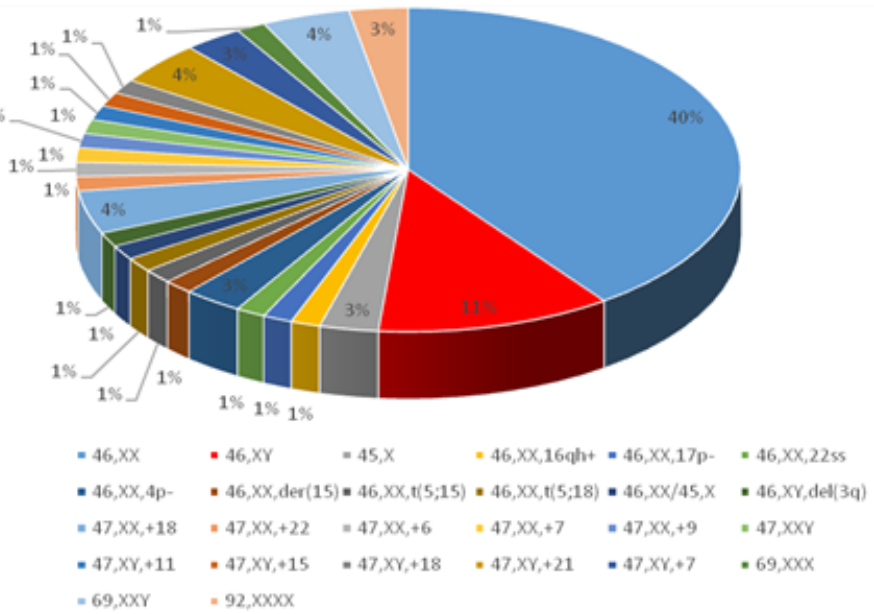

Fig. 3. Karyotype founded in the study lot

The patients were classified in two groups, group 1 included patients $<35$ and group 2 included patients $\geq 35$ years. When comparing the gestational age between the two groups, statistical significant deference, was observed. The median gestation age in group 1 being significantly lower than in group $2(\mathrm{P}<0.0001)$ (fig. 4).

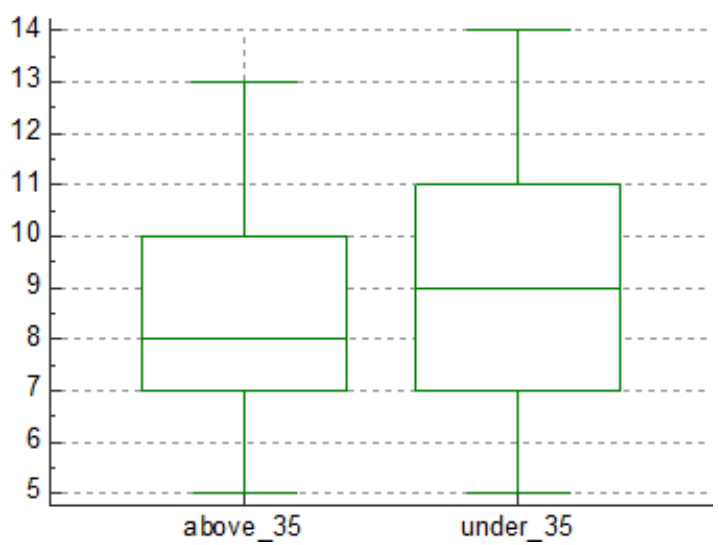

Fig. 4. Gestational age in patients $<35$ and patients $\geq 35$ years

A higher incidence of chromosomal anomalies was observed in the group 2 including patients $\geq 35$ years $(55.5 \%$ vs $45.5 \%)$. In the patients aged $<35$ years was 
detected abnormal karyotype in 24 out of 52 patients $(45.5 \%)$, the most frequent were the trisomies $(45.83 \%)$, followed by structural aberrations (29.16\%), triploidies $(12.5 \%), 2$ cases $(8,33 \%)$ of presented monosomy and one case $(4.16 \%)$ was with tetraplody. In the patients aged $\geq 35$ years was detected abnormal karyotype in 10 out of 18 patients $(55.5 \%)$, the most frequent were the trisomies $(50 \%)$, followed by structural aberrations ( $20 \%)$, followed by mosaic monosomy, triploidy and tetraplody (10\%) (fig. 5).

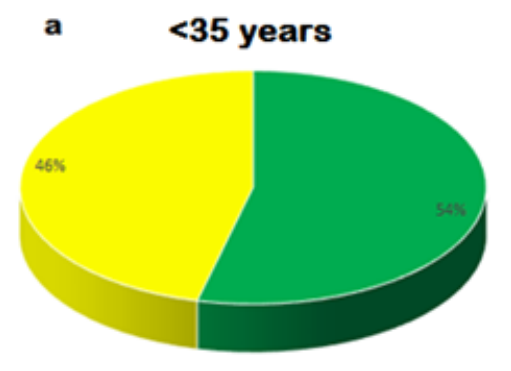

Thormal kanotipe

b $\geq 35$ years

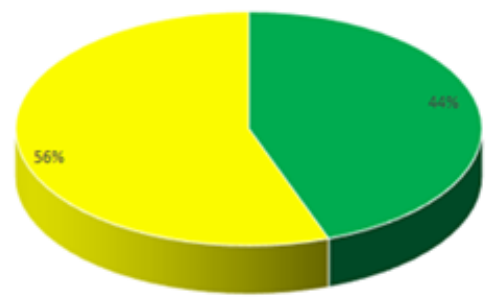

abnormal kanotipe

The incidence of chromosomal anomalies for each maternal age is presented in figure 6 , showing higher rate of chromosome imbalances on the patients $\geq 35$ years old.

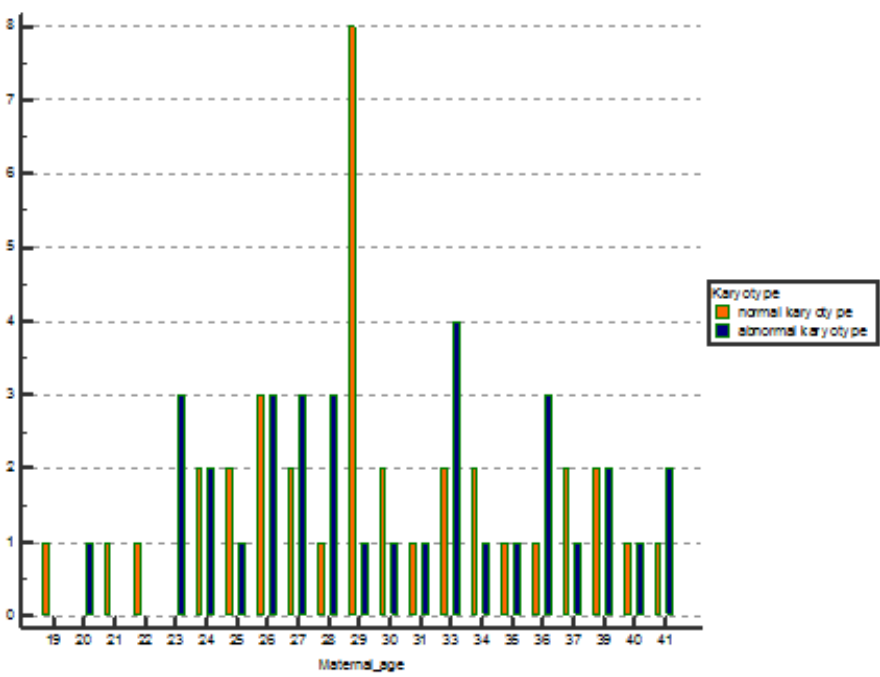

Fig. 6. Incidence of chromosomal anomalies for each maternal age

We evaluated the age of the aborted fetus in correlation with the karyotype.

We established the following groups: miscarriages with abnormal karyotype at $\leq 7$ and $>7$ weeks of gestation and fetuses with normal karyotype at $\leq 7$ and $>7$ weeks of gestation.

At $\leq 7$ weeks of gestation in the $<35$ years' subgroups, 4 of 14 miscarriages (28.57\%) presented chromosomal abnormalities, while in the $\geq 35$ years' subgroups, 3 of 5 miscarriages $(60 \%)$ were cytogenetically abnormal. At > 7 weeks of gestation in the $<35$ years' subgroups, 20 of 38 miscarriages(52.63\%) presented chromosomal abnormalities, while in the $\geq 35$ years' subgroups, 7 of 13 miscarriages (53.84\%) were cytogenetically abnormal.

Many time is difficult to establish the cause of pregnancy loss, but the information is very important for the couple counselling. For an important percent of the miscarriages, chromosomal aberrations are the most probable cause of pregnancy loss [12-14].

Regarding the rate of chromosomal abnormalities in spontaneous abortions, the data varies between 50 to $80 \%$ for first trimester miscarriages but there are several factors that may influence the rate of chromosomal aberrations as the selection bias of the cases for which cytogenetic analysis is done, the gestational age distribution, the maternal age distribution, the tissue samples analyzed, the expertise in cytogenetic methods [18-20].

It is known that chromosomal aberrations are due to errors in gametogenesis as well as in fertilization and mitotic division after fertilization [6]. In the study lot the incidence of chromosomal aberrations was $48.57 \%$ including errors due to all of the mechanism describe above: monosomies, trisomies, triploidy, tetraploidy, structural aberrations.

The incidence of chromosomal aberrations is in line with other reports regarding chromosomal imbalances in aborted fetuses [21, 22]. As reported we take in consideration a possible contamination with maternal cell due to a higher rate of normal female karyotype as compared with normal male karyotypes (28/70 were $46, X X$, while only $8 / 70$ were $46, X Y$ ). This is in line with literature data showing that 30 to $40 \%$ of the normal female karyotype obtained by conventional cytogenetics are of maternal origin $[23,24]$.

The relative frequency of specific chromosome anomalies was: $47.05 \%$ for trisomy, $8.82 \%$ for monosomy $X, 11.76 \%$ for triploidy, $5.88 \%$ for tetraploidy and $26.47 \%$ for structural rearrangements. The rate for chromosomal rearrangements in the present study is higher than the data found in other reports published $[22,25,26]$. As there is a high probability of recurrence for the structural rearrangement the genetic counselling of the couple was done and we recommended the cytogenetic evaluation of the genitors in order to have a proper management of a future pregnancy. It was found that after a miscarriage with trisomic fetus, there is an increased risk for other trisomies in the future pregnancies [27-29]. Regarding the recurrence risk of a miscarriage was found that there is a lower risk if the fetus has a chromosome abnormality [ 1 , 28].

It is known that a significant risk factor for nondisjunction in oogenesis is the maternal age $\geq 35$ years. (26) In the present study we observed a statistic significant difference between the chromosomal rate of the patient group including females under 35 years old (45.5\%) and the group of patients aged $\geq 35$ years ( $55.5 \%$ ).

A significant difference was observed when comparing the cytogenetic results of the aborted fetuses having $\leq 7$ and $>7$ weeks of gestation in correlation with maternal age. If the chromosomal aberration rate for the patients aged $\geq 35$ years was almost similar for fetuses having $\leq 7$ weeks of gestation and those having $>7$ weeks of gestation, in the patients aged $<35$ years, there was a significant increase of the miscarriage rate after 7 weeks of gestation (52.63\%) when compared with the miscarriage rate under 7 weeks of gestation (28.57\%).

In our study lot, 15 aborted fetuses (21.42\%) exhibit a chromosomal aberration that did not involve chromosome $13,18,21, X$ nor $Y$, chromosome that can be evaluated by rapid methods including FISH or QF-PCR. If using one of 
the rapid methods, almost a quarter of the patients would not have identified the cause of the miscarriage.

The information on products of conception given by the karyotype is very useful for both physicians and patients [18]. It is also useful for the further genetic and psychological counselling.

It has been stated that miscarriages, whether sporadic or recurrent, often result in high levels of emotional and psychological distress. Miscarriages are a traumatic experience with a high degree of psychological implication.

Typically, a miscarriage may cause dysphoria and grief, and therefore, depression. Also, given the sudden and unexpected nature of the situation, patients may also experience high anxiety levels [15].

According to the literature, miscarriages further represent a significant emotional burden not only for women but also for their male partners. This is why, also the partners must be implied in the further psychological counseling and psychotherapy. Through the psychological counselling and psychotherapy sessions, the patients can better manage the whole life situation and their functioning and life quality will be improved on all domains. Also the further development of psychopathology, meaning anxiety, depressive disorders or even psychosis, through better management of these life stressors and events $[16,17]$.

Clinicians should screen women frequently for depressive and anxiety symptoms after a miscarriage. So that psychological counselling, in addition to medical investigations and consultation, is beneficial in reducing women's distress after miscarriage [15]. Absence of an identifiable cause led to maintenance of the initial levels of anxiety, which could have decreased otherwise in time. Even if the symptoms improve and women do get pregnant again with proper counselling, mood disturbances can still coexist after several months.

Interestingly, this emotional distress can be better managed if the cause of miscarriage is provided. Stress and feelings of self-blame, guilt and anxiety diminish when information about the cause of miscarriage, mainly karyotype, is provided. Women are seeking for answers: as evidenced in a survey published, where although $7 \%$ of them were offered chromosome testing in a first miscarriage, they would all have it done again in a future miscarriage. Moreover, $65 \%$ of those who did not have this test performed, wished they could have had the test done [15-17].

\section{Conclusions}

Cytogenetic analysis in aborted fetuses provide important information for establishing the risk of recurrence of chromosome imbalances in subsequent pregnancies for patients with spontaneous abortions. The results of cytogenetic analysis of first trimester miscarriage is an important tool that can enhance diagnostic evaluation, and represent a critical information for counselling infertile couples.

Post-miscarriage genetic and psychological counselling and appropriate treatment are crucial for the prevention of high psychological distress, psychiatric symptoms and psychopathology in these patients. Proper management of depressive and anxiety symptoms after pregnancy loss can highly improve the quality of life of the patients and also their families.

Acknowledgments. This research was partially done in the Center of Genomic Medicine from the Victor Babes University of Medicine and Pharmacy, Timisoara, POSCCE ProjectID: 1854, cod SMIS: 48749, Center of Genomic Medicine v2, contract 677/09.04.2015.

\section{References}

1. STEPHENSON MD. Frequency of factors associated with habitual abortion in 197 couples. Fertil Steril, 1996, 66(1):24-33.

2. FORD HB, SCHUST DJ. Recurrent pregnancy loss: etiology, diagnosis, and therapy. Rev Obstet Gynecol, 2009, 2(2):76-83.

3.LJUNGER E, CNATTINGIUSS, LUNDIN C, ANNEREN G. Chromosomal anomalies in first-trimester miscarriages. Acta Obstet Gynecol Scand, 2005, 84(11):1103-1110.

4.YUSUF RZ, NAEEM R. Cytogenetic abnormalities in products of conception: a relationship revisited. Am J Reprod Immunol N Y N, 2004, 52(1):88-96.

5.MORALES C, SANCHEZ A, BRUGUERA J, MARGARIT E, BORRELL A, BOROBIO V, et al. Cytogenetic study of spontaneous abortions using semi-direct analysis of chorionic villi samples detects the broadest spectrum of chromosome abnormalities. Am J Med Genet A, 2008, 146A(1):66-70.

6.PENDINA AA, EFIMOVA OA, CHIRYAEVA OG, TIKHONOV AV, PETROVA LI, DUDKINA VS, et al. A comparative cytogenetic study of miscarriages after IVF and natural conception in women aged under and over 35 years. J Assist Reprod Genet, 2014, 31(2):149-204.

7.MORTON NE, CHIU D, HOLLAND C, JACOBS PA, PETTAY D. Chromosome anomalies as predictors of recurrence risk for spontaneous abortion. Am J Med Genet, 1987, 28(2):353-413.

8.** Practice Committee of the American Society for Reproductive Medicine. Evaluation and treatment of recurrent pregnancy loss: a committee opinion. Fertil Steril, 2012, 98(5):1103-1114.

9.J ASLOW CR, CARNEY JL, KUTTEH WH. Diagnostic factors identified in 1020 women with two versus three or more recurrent pregnancy losses. Fertil Steril, 2010, 93(4):1234-1277.

10.GUERNERI S, BETTIO D, SIMONI G, BRAMBATI B, LANZANI A, FRACCARO M. Prevalence and distribution of chromosome abnormalities in a sample of first trimester internal abortions. Hum Reprod Oxf Engl, 1987, 2(8):735-744.

11.EIBEN B, BARTELS I, BÄHR-PORSCH S, BORGMANN S, GATZ G, GELLERT G, et al. Cytogenetic analysis of 750 spontaneous abortions with the direct-preparation method of chorionic villi and its implications for studying genetic causes of pregnancy wastage. Am J Hum Genet, 1990, 47(4):656-719.

12.MARQUARD K, WESTPHAL LM, MILKI AA, LATHI RB. Etiology of recurrent pregnancy loss in women over the age of 35 years. Fertil Steril, 2010, 94(4):1473-1480.

13.HODES-WERTZ B, GRIFO J, GHADIR S, KAPLAN B, LASKIN CA, GLASSNER $M$, et al. Idiopathic recurrent miscarriage is caused mostly by aneuploid embryos. Fertil Steril, 2012, 98(3):675-680.

14.RAI R, REGAN L. Recurrent miscarriage. Lancet Lond Engl, 2006, 368(9535):601-612.

15.NIKCEVIC AV, KUCZMIERCZYK AR, NICOLAIDES KH. The influence of medical and psychological interventions on women's distress after miscarriage. J Psychosomat Research, 2007, 63(3): 283-290.

16.NYNAS J, NARANG P, KOLIKONDA MK, LIPPMANN S. Depression and anxiety following early pregnancy loss: recommendations for primary care providers. Prim Care Companion CNS Disord, 2015, 17(1):408-418.

17.CHIRIAC DV, HOGEA LM, BREDICEAN AC, REDNIC R, NUSSBAUM LA, HOGEA GB, GRIGORAS ML. A rare case of Meckel-Gruber syndrome. Rom J Morphol Embryol, 2017, 58(3):1023-1027.

18.PHILIPP T, KALOUSEK DK. Generalized abnormal embryonic development in missed abortion: embryoscopic and cytogenetic findings. Am J Med Genet, 2002, 111(1):43-50.

19.PETRACCHI F, COLACI DS, IGARZABAL L, GADOW E. Cytogenetic analysis of first trimester pregnancy loss. Int J Gynaecol Obstet Off Organ Int Fed Gynaecol Obstet, 2009, 104(3):243-257.

20.J OBANPUTRA V, ESTEVES C, SOBRINO A, BROWN S, KLINE J, WARBURTON D. Using FISH to increase the yield and accuracy of karyotypes from spontaneous abortion specimens. Prenat Diagn, 2011, 31(8):755-764. 
21.CHOI TY, LEE HM, PARK WK, JEONG SY, MOON HS. Spontaneous abortion and recurrent miscarriage: A comparison of cytogenetic diagnosis in 250 cases. Obstet Gynecol Sci, 2014, 57(6):518-525.

22.J ENDERNY J. Chromosome aberrations in a large series of spontaneous miscarriages in the German population and review of the literature. Mol Cytogenet, 2014, 53(4):38-45.

23.KARAOGUZ MY, NAST, KONAC E, INCE D, PALA E, MENEVSE S. IS cytogenetic diagnosis of $46, X X$ karyotype spontaneous abortion specimens erroneous? Fluorescence in situ hybridization as a confirmatory technique. J Obstet Gynaecol Res, 2005, 31(6):508-513. 24.J ARRETT KL, MICHAELIS RC, PHELAN MC, VINCENT VA, BEST RG. Microsatellite analysis reveals a high incidence of maternal cell contamination in $46, X X$ products of conception consisting of villi or a combination of villi and membranous material. Am J Obstet Gynecol, 2001,185(1):198-203.
25.BASTOS R, RAMALHO C, DORIA S. Prevalence of chromosomal abnormalities in spontaneous abortions or fetal deaths. Acta Med Port, 2014, 27(1):42-50.

26.GODDIJ N M, LESCHOT NJ . Genetic aspects of miscarriage. Baillieres Best Pract Res Clin Obstet Gynaecol, 2000, 14(5):855-865.

27.WARBURTON D, DALLAIRE L, THANGAVELU M, ROSS L, LEVIN B, KLINE J. Trisomy recurrence: a reconsideration based on North American data. Am J Hum Genet, 2004, 75(3):376-385.

28.CARP H, TODER V, AVIRAM A, DANIELY M, MASHIACH S, BARKAI G. Karyotype of the abortus in recurrent miscarriage. Fertil Steril. 2001, 75(4):678-682.

29.HASSOLD T, HUNT P. Maternal age and chromosomally abnormal pregnancies: what we know and what we wish we knew. Curr Opin Pediatr, 2009, 21(6):703-711.

Manuscript received: 3.08 .2018 